\title{
Fundamental study for estimating azimuthal shear wave anisotropy by applying VSM in marine airgun survey
}

\author{
Yusuke WATANABE $^{1}$, Hitoshi MIKADA ${ }^{1}$ and Junichi TAKEKAWA ${ }^{1}$ \\ ${ }^{1}$ Dept. of Civil and Earth Res. Eng., Kyoto University
}

\begin{abstract}
In this study we utilize the virtual source method to overcome existing problems surrounding marine shear wave survey. We improve the virtual source method (VSM) to enable to generate shear wave signal even in marine survey by using airgun records and a single ocean bottom seismometer (OBS). To evaluate this method, we conduct numerical experiments by using 3D finite difference simulation including an azimuthal anisotropic layer. We generate virtual 4C data by using our VSM and estimate azimuthal direction in subsurface of the model by applying the Alford rotation to these virtual $4 \mathrm{C}$ data with reasonable accuracy.
\end{abstract}

\section{INTRODUCTION}

Shear wave survey takes more and more important role because it gives information of not only geometrical structure of subsurface but also lithological properties. Although shear wave survey has these advantages, it is difficult to use it in marine environment because shear modulus of water is zero. In recent year, some researchers proposed signal processing method to emphasize shear wave signal ${ }^{1)}$. They applied the virtual source method (VSM) to received data. They demonstrated how effective their method was in numerical simulation or laboratory experiments, but more detail data processing by utilizing those data has not been done efficiently.

In this paper, we put forward new application of the virtual source method (VSM) ${ }^{2)}$ to received data by ocean bottom seismometer (OBS) for estimating azimuthal anisotropic structure in subsurface. The VSM enable us to generate virtual traces from data sets of an array of air-gun shots and single OBS on the sea floor. After that we apply the Alford rotation $^{3)}$ to virtual traces in order to estimate azimuthal anisotropic structure in subsurface.

To evaluate the effectiveness of our method, we use synthetic seismic data sets from 3D-finite difference seismic simulation with rotated staggered $\operatorname{grid}^{4)}$. We put a line of the explosive source in the sea water, and a single OBS on the seafloor of the model. We put an anisotropic and isotropic layer in the model. We set some different azimuthal angle to anisotropic layer to test the accuracy of our method.

\section{3D SYNTHETIC DATA}

In this chapter, we present the method to make synthetic 3D seismic data and the shot gathers.

\section{(1)Methods}

As shown in Figure 1, we use a simple 5 layered model. We put OBS on the seafloor in the center of the model. We define a $1200 \mathrm{~m}$ length of survey line and we put 101 air-gun sources in every $12 \mathrm{~m}$ near the sea surface. The source function is the Ricker wavelet with $10 \mathrm{~Hz}$ peak frequency. We use PML absorbing layer at the end of the model in order to absorb artificial reflection waves. We modeled the anisotropic layer as HTI model with azimuthal angle of 45 and 60 degrees. We define these models as 45-degree model and 60-degree model. Model properties and elements of stiffness matrix are shown in Table1 and Table 2.

Table 1 Parameters of the model

\begin{tabular}{|c|c|c|c|c|c|}
\hline \multicolumn{2}{|c|}{ Sea layer } & \multicolumn{2}{|c|}{ Iso layer 1} & \multicolumn{2}{|c|}{ Aniso layer 2} \\
\hline$\rho_{W}\left(\mathrm{~kg} / \mathrm{m}^{3}\right)$ & 1000 & $\rho_{1}\left(\mathrm{~kg} / \mathrm{m}^{\prime}\right)$ & 2000 & $\rho_{2}\left(\mathrm{~kg} / \mathrm{m}^{\mathrm{\prime}}\right)$ & 2200 \\
\hline$V_{p}(m / s)$ & 1500 & $V_{p}(m / s)$ & 1800 & $V_{p f}(m / s) 2000$ & $V_{s f}^{(m / s)} 1155$ \\
\hline$V_{s}(m / s)$ & $\mathbf{0}$ & $V_{s}(m / s)$ & 1039 & $V_{p s}(m / s) 1900$ & $V_{\text {ss }}(\mathrm{m} / \mathrm{s}) \quad 1039$ \\
\hline \multicolumn{2}{|c|}{ Iso layer 3} & \multicolumn{2}{|c|}{ Iso layer 4} & \multicolumn{2}{|c|}{ Another parameter } \\
\hline$\rho_{3}\left(\mathrm{~kg} / \mathrm{m}^{3}\right)$ & 2300 & $\rho_{2}\left(\mathrm{~kg} / \mathrm{m}^{\mathrm{m}}\right)$ & 2500 & $\Delta \mathbf{x}(\mathrm{m})$ & 3.0 \\
\hline \multirow{2}{*}{$V_{p}(m / s)$} & \multirow{2}{*}{2200} & \multirow{2}{*}{$V_{p}(m / s)$} & \multirow{2}{*}{2500} & $\Delta t_{\text {(ms) }}$ & 0.25 \\
\hline & & & & Timesteps & 16384 \\
\hline \multirow{2}{*}{$\mathbf{V}_{\mathbf{s}}(\mathbf{m} / \mathbf{s})$} & \multirow{2}{*}{1270} & \multirow{2}{*}{$V_{s}(\mathbf{m} / s)$} & \multirow{2}{*}{1443} & Survey time & 4.09575 \\
\hline & & & & $f_{\text {peak }}$ & 10.0 \\
\hline
\end{tabular}


Table2 Values of stiffness tensor in anisotropic layer

\begin{tabular}{|c|c|c|c|}
\hline \multicolumn{4}{|c|}{ Stiffness value [GPa] } \\
\hline $\mathrm{C}_{11}$ & 8.8 & $\mathrm{C}_{44}$ & 2.376 \\
\hline $\mathrm{C}_{33}$ & 7.942 & $\mathrm{C}_{66}$ & 2.933 \\
\hline $\mathrm{C}_{13}$ & 2.701 & $\mathrm{C}_{33}-2 \mathrm{C}_{44}$ & 3.19 \\
\hline
\end{tabular}

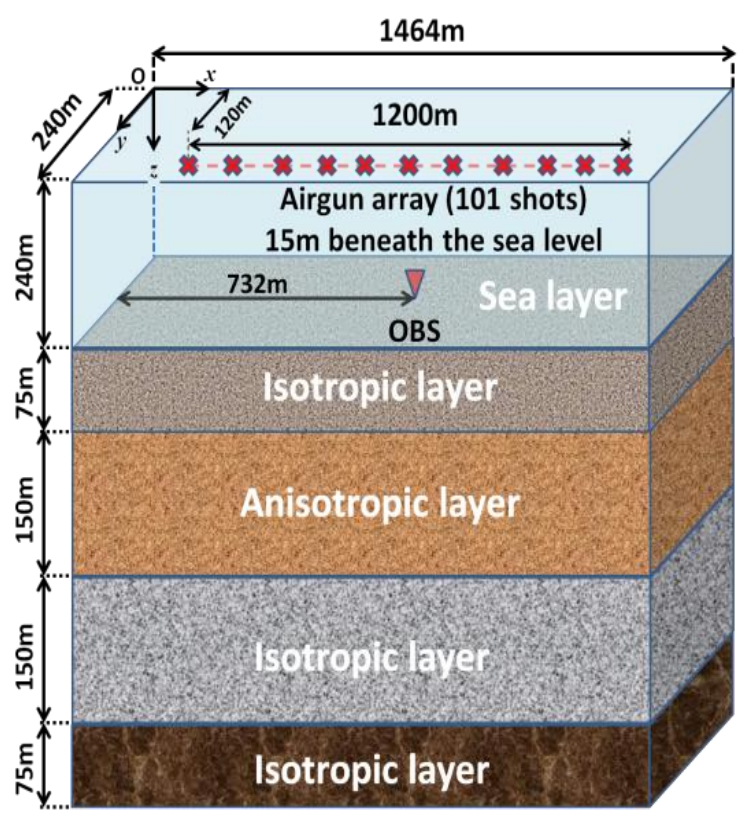

Figure 1 The specifications of the model

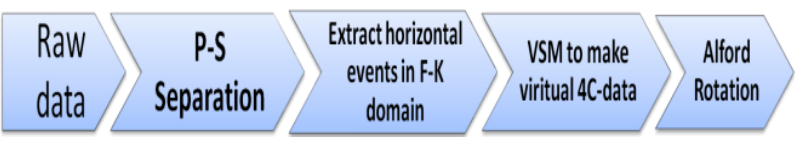

Figure 3 Processing flow chart

\section{(2)Shot gathers}

Figure 2 is shot gathers of each model and direction. The left column is 45-degree model, and the right column is 60 -degree model. The range of amplitude is $\pm 1 e^{-5}$ in $x$ and $z$ direction, and $\pm 3 e^{-6}$ in $y$ direction.
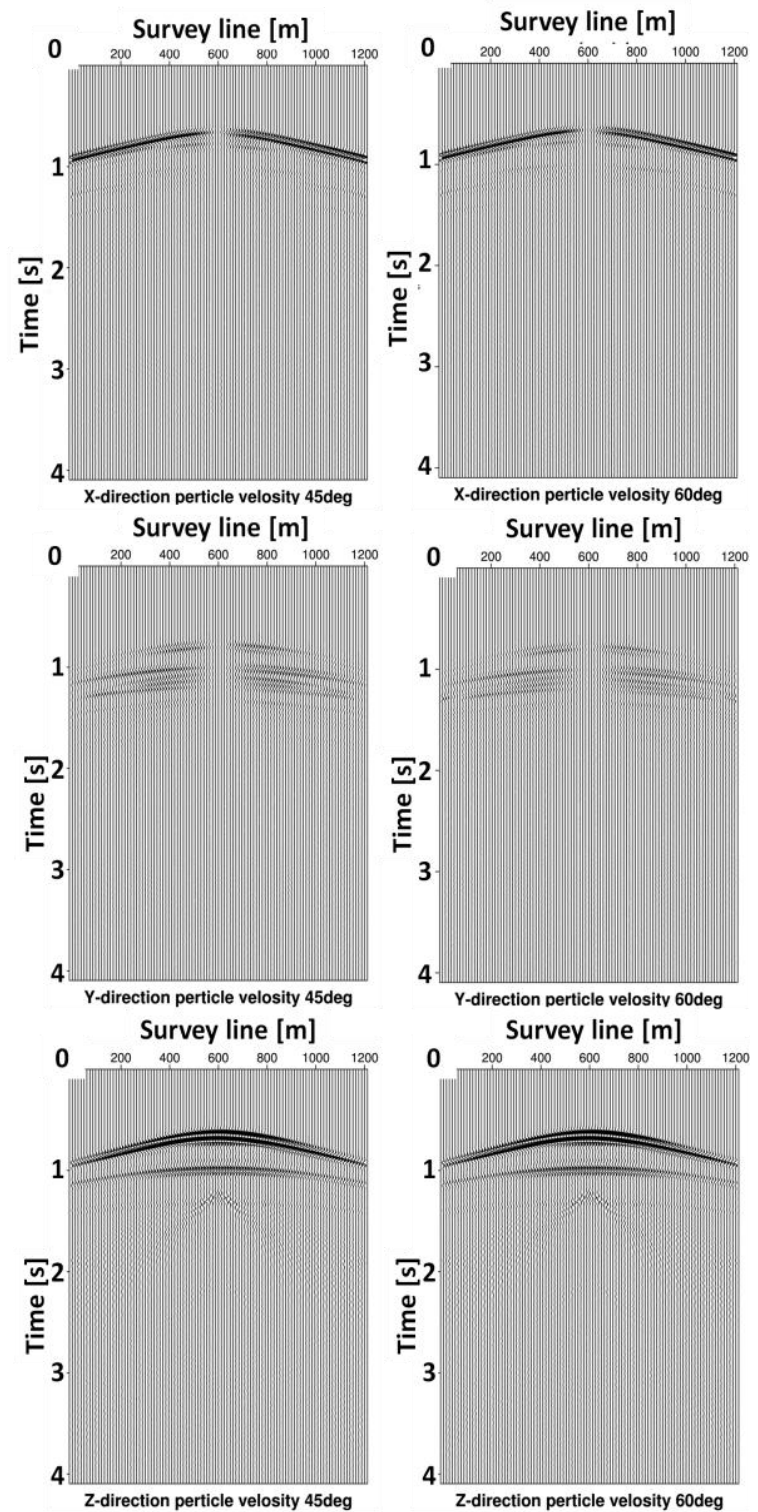

Figure 2 Shot gathers of each model

\section{VIRTUAL 4C DATA}

We show the method of synthesizing virtual 4C data and azimuthal anisotropic angle estimation in Figure 3.

\section{(1) P-S wave field separation}

As first step of the processing, we do P-S wave field separation ${ }^{5)}$ to the reflection signal in order to emphasis PS converted signal from the subsurface. We do this method to the received data in $x$-direction and we use $\mathrm{S}$ wave field data in $x$-direction for later processing. 


\section{(2) Extract horizontal events in F-K domain}

After P-S wave field separation, we extract horizontal events in F-K domain because we now assume the model as horizontally layered structure. So the reflection signal should have small wave number in F-K domain. Hence we extract the event which contains less than $5 \Delta k(1 / \mathrm{m})$ in $x$-direction and $y$-direction. $\Delta k$ means the step size of wave number.
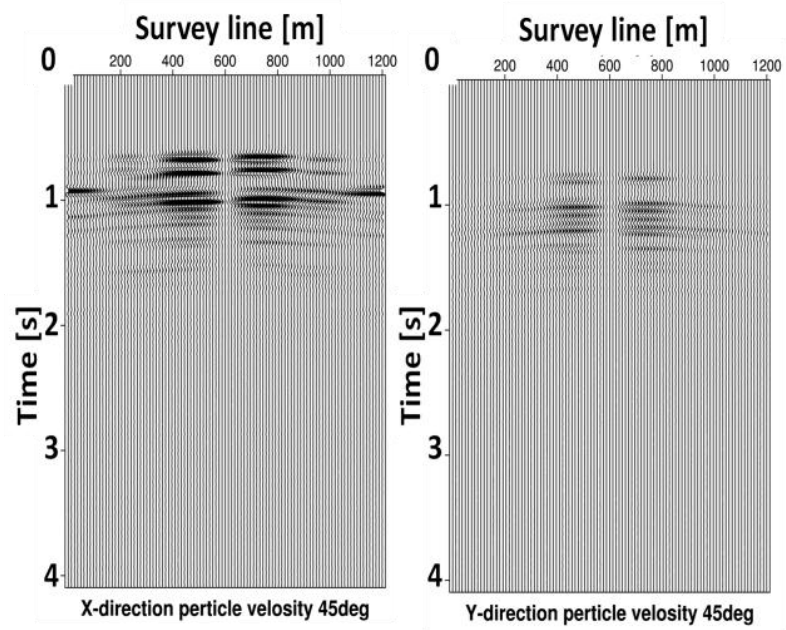

Figure 4 Processed shot gather in $45 \mathrm{deg}$ model
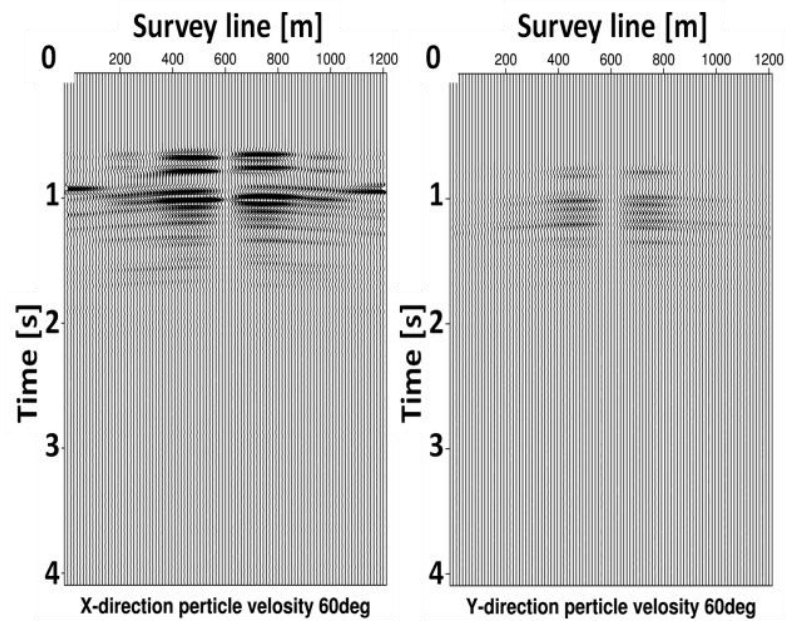

Figure 5 Processed shot gather in 60deg model

Figure 4 and Figure 5 are the processed data in each model. The range of amplitude is $\pm 1 e^{-6}$.

\section{(3) VSM to make virtual 4C data}

Now we make virtual 4C data by using VSM. As shown in Figure 6, we can see virtual shot record at each shot position by taking cross-correlation to each neighboring shot record. There are totally 101 shot records, so we can make 100 virtual traces in each component. For example, we take two pairs L1-L2 and R1-R2. In the case of the left side from OBS, L1-L2, we use these equations below.

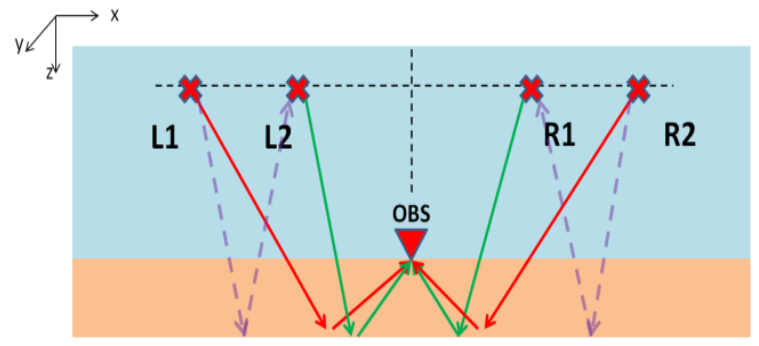

Figure 6 Image of VSM

$$
\begin{array}{ll}
V_{x x}=V_{L 1 x} \otimes V_{L 2 x} & V_{x y}=V_{L 1 x} \otimes V_{L 2 y} \\
V_{y x}=V_{L 1 y} \otimes V_{L 2 x} & V_{y y}=V_{L 1 y} \otimes V_{L 2 y}
\end{array}
$$

$V_{L 1 x}$ and $V_{L 1 y}$ means true received data of $x$ - or $y$-direction particle velocity from L1 or L2. $\otimes$ means cross-correlation, $V_{x x}$ means virtually shots in $x$ direction at $\mathrm{L} 1$ or $\mathrm{R} 1$ and virtually receives in $x$ direction at L2 or R2. In the case of R1-R2, we use the equations similar to the L1-L2 case.

$$
\begin{array}{ll}
V_{x x}=V_{R 2 x} \otimes V_{R 1 x} & V_{x y}=V_{R 2 x} \otimes V_{R 1 y} \\
V_{y x}=V_{R 2 y} \otimes V_{R 1 x} & V_{y y}=V_{R 2 y} \otimes V_{R 1 y}
\end{array}
$$

By using equations (1) and (2), we can extract virtual shear signals from air-gun data. In other words, we can extract horizontal forces from explosive forces just correlating neighboring traces (red and green arrows in Figure 6). Through these processing, we can create virtual common offset gathers (blue dot arrow in Figure 6).

\section{(4) Alford rotation}

Finally we estimate azimuthal angle by applying Alford rotation to these virtual data.

\section{RESULTS AND DISCUSSIONS}

In this section, we'll show the result of virtual 4C data and the final results after applying Alford rotation in each model.

\section{(1) Virtual 4C data sets}

Figure $7-\mathbf{1 4}$ are the virtual $4 \mathrm{C}$ gathers in each models. To emphasis the shear signal, the direct wave and the reflection waves from the seafloor are muted in these figures $(0-0.3 \mathrm{~s})$. 


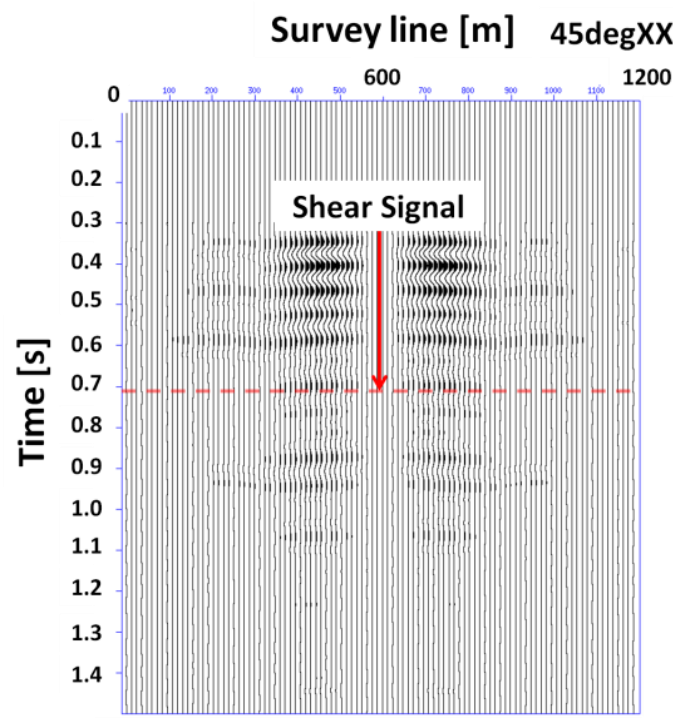

Figure 7 Virtual 4C gather in 45deg. $x x$

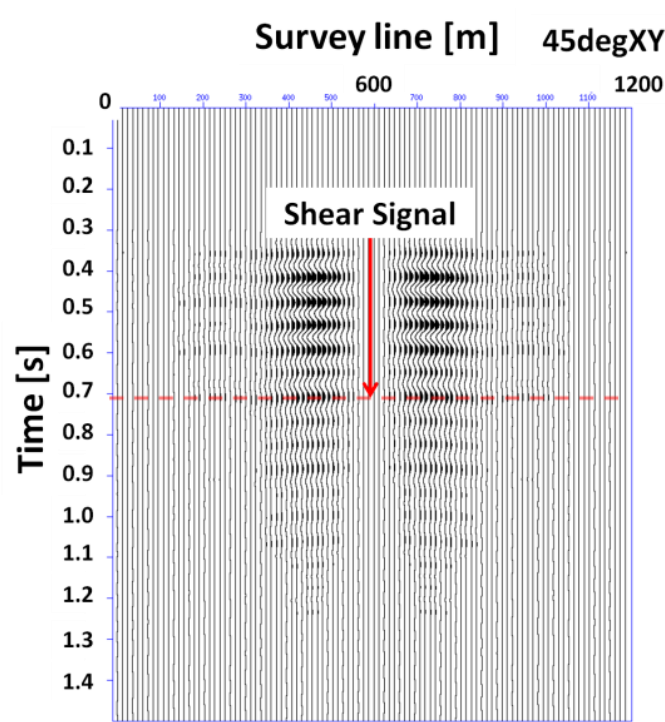

Figure 8 Virtual $4 \mathrm{C}$ gather in $45 \mathrm{deg}$. $x y$

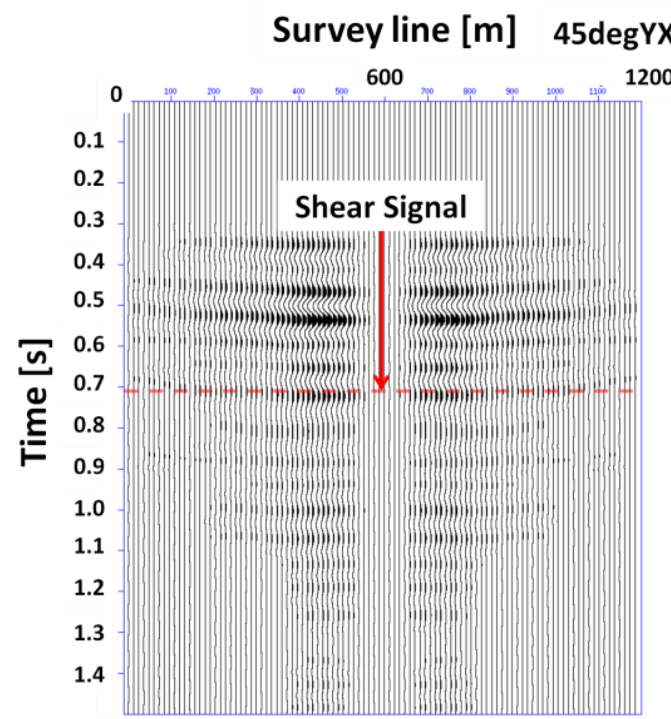

Figure 9 Virtual 4C gather in 45deg. $y x$

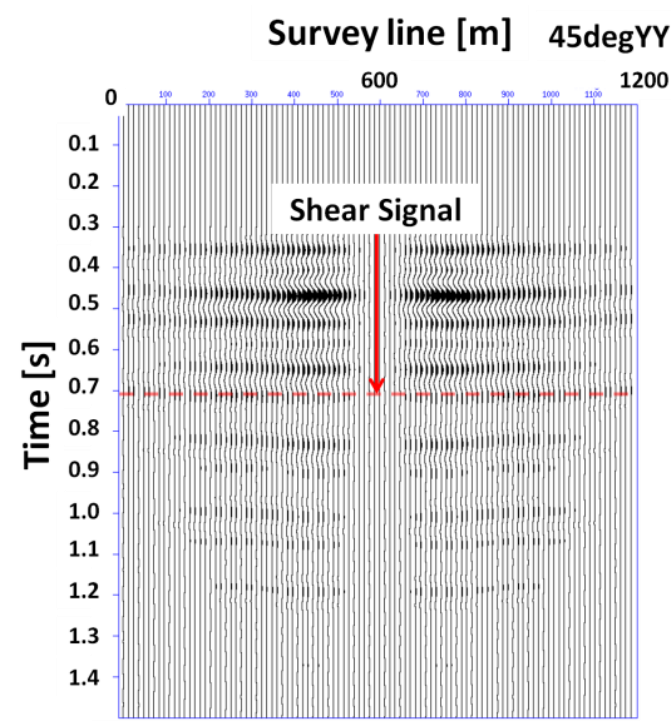

Figure 10 Virtual 4C gather in $45 \mathrm{deg}$. $y y$

Survey line $[\mathrm{m}]$ 60degXX

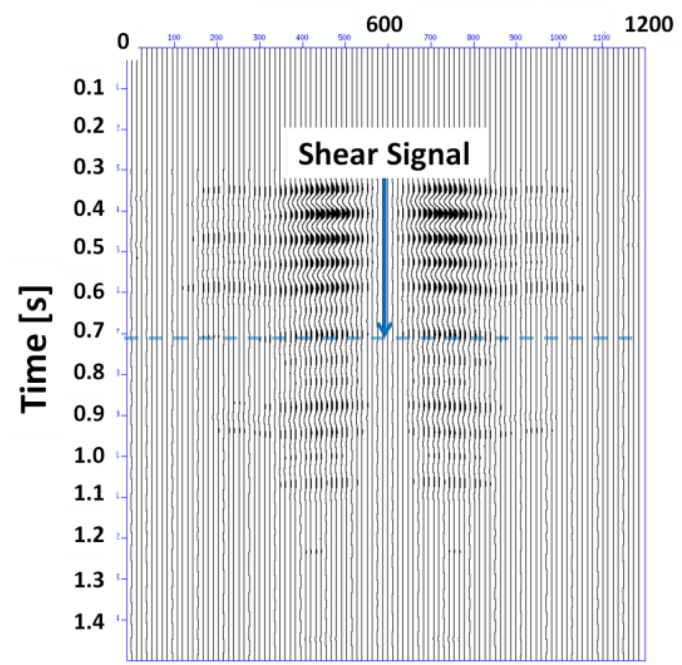

Figure 11 Virtual 4C gather in 60deg. $x x$

Survey line [m] 60degXY

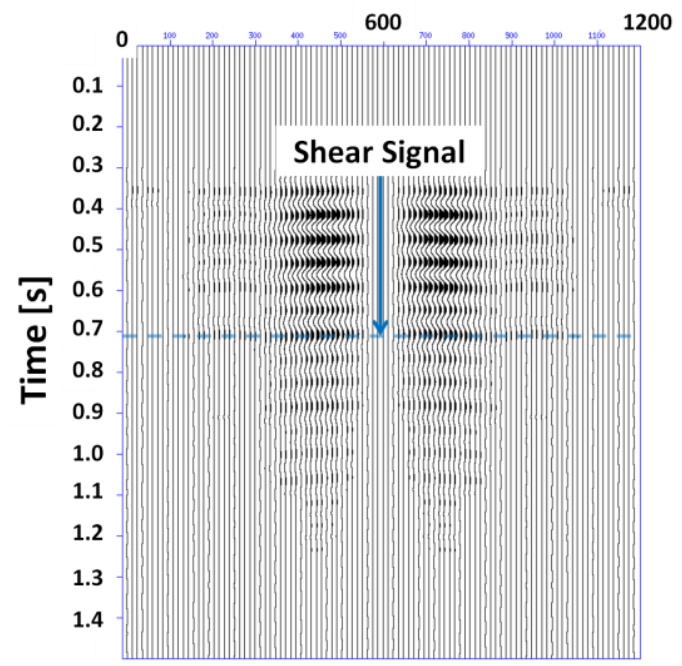

Figure 12 Virtual $4 \mathrm{C}$ gather in 60deg. $x y$ 


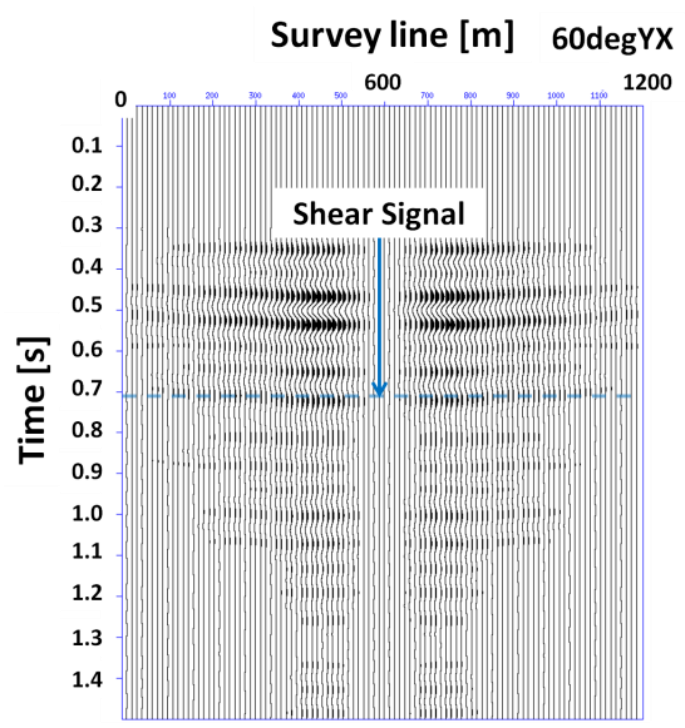

Figure 13 Virtual 4C gather in 60deg. $y x$

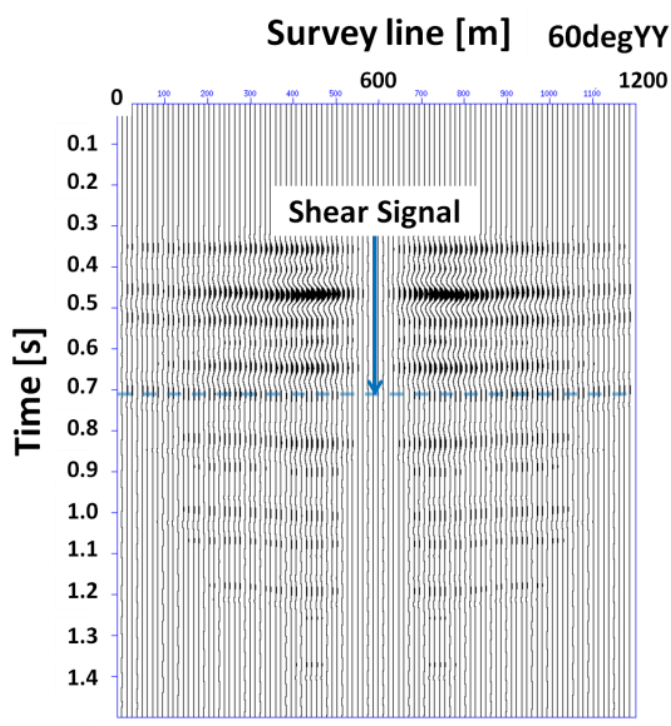

Figure 14 Virtual 4C gather in 60deg. yy

Considering virtual source-receiver geometry, we can see the P-S-S-P signal from the bottom of the anisotropic layer around $0.7 \mathrm{sec}$ in theoretical travel time.

\section{(2) Estimated angle after Alford rotation}

In this section, we'll show the final result after Alford rotation. We estimate azimuthal angle in the model by using Alford rotation. We applied this method to the VSM data. We showed last section in $0.6-0.9 \mathrm{~s}$. The results for 45-degree model and 60-degree model are shown in Figure 15 and16. In these Figures, $x$-axis means the placement of the survey line and $y$-axis means the estimated azimuthal anisotropic angle. The shaded line means the true value of the model.

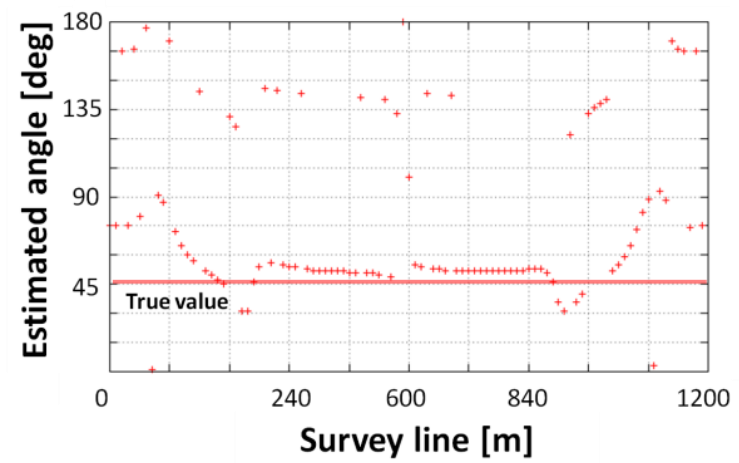

Figure 15 Estimated angle in 45-deg. model

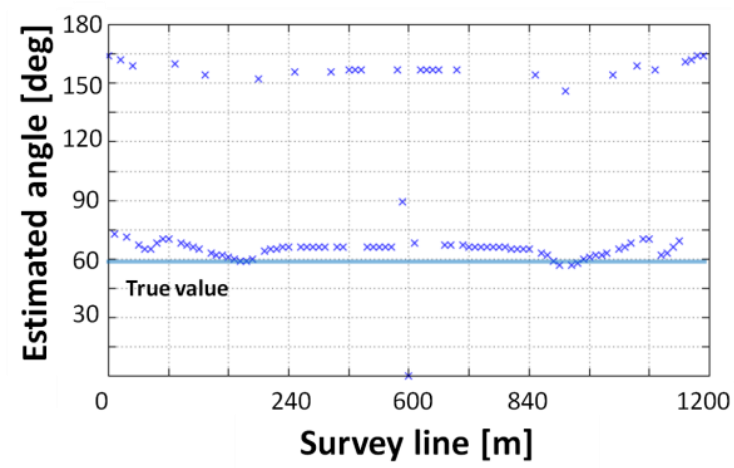

Figure 16 Estimated angle in 60-deg. model

As shown in Figure 15 and 16, we could estimate the azimuthal angle with reasonable accuracy near the OBS, 240-840m. This is because we couldn't utilize the energy in high order Fresnel zone, we couldn't gain sufficient $\mathrm{S} / \mathrm{N}$ ratio around the end of survey line. Another reason of the error comes from the contamination of the signal from other layers.

\section{CONCLUSION}

We applied VSM to 3D synthetic data recorded by a single OBS and a single airgun survey line so as to gain the information of the subsurface anisotropic layer. To evaluate the effectiveness of this method, we carried out the numerical 3D seismic simulation with an anisotropic layer. With these data, we made the virtual $4 \mathrm{C}$ data. After that we estimated azimuthal angle of the anisotropic layer by applying the Alford rotation to the virtual 4C data. The results of our numerical experiments show that this method can estimate the azimuthal angle in anisotropic layer though the use of airgun sources in marine environment. From this result, we could get not only lithological properties but also the distribution or density of existing cracks in subsurface.

ACKNOWLEDGMENT: We use SU for making figures, so we would like to thank staffs of CWP. 


\section{REFERENCES}

1) Alford, R.M., 1986, Shear Data in the Presence of Azimuthal Anisotropy: $56^{\text {th }}$ Annual meeting, Society of Exploration Geophysicists, Expand Abstracts, 476-479.

2) Bakulin, A., Calvert, R., Shell International E\&P, 2004, Virtual Source: new method for imaging and 4D below complex overburden: $74^{\text {th }}$ Annual International Meeting, SEG, Expanded Abstracts, 1921-1924.

3) Bakulin, A., Calvert, R., 2006, The virtual source method: Theory and case study: Geophysics, 71, SI139-SI150.

4) Bakulin, A., Calvert, R., Shell International E\&P, 2005, Virtual shear source: A new methods for shear-wave seismic surveys: $75^{\text {th }}$ Annual International Meeting, SEG, Expanded Abstracts, 2633-2636.

5) Bakulin, A., Mateeva, A., Calvert, R., Jorgensen, P., Lopez, J., 2007, Virtual shear source makes shear waves with air guns: Geophysics, 72, P. A7-A11.

6) Bakulin, A., Mateeva, A., 2008, Estimating interval shear-wave splitting from multicomponent virtual shear check shots: Geophysics, 73,

P.A39-A43

7) Collino, F., Tsogka, C., 2001, Application of the perfectly matched absorbing layer model to the linear elastodynamic problem in anisotropic heterogenous media: Geophysics, 66, P.294-307. 8) Devaney, A.J., Oristaglio, M.L., 1986, A plane-wave decomposition for elastic wave fields applied to the separation of $\mathrm{P}$-waves and $\mathrm{S}$-waves In vector seismic data: Geophysics, 51, P. 419-423. 9) Nagai,Y., Tsuda, A., Ozasa H., Hatanaka,H., Tanaka, K., Tagami, M., Sato, F., Takekawa, J., Mikada, H., 2016, Towed Marine Dipole Source for Shear Wave Generation: $78^{\text {th }}$ EAGE Conference and Exhibition

10) Saenger, E.H., Gold, N., Shapiro, A., 2000, Modeling the propagation of elastic waves using a modified finite-difference grid: Wave Motion, 31, 77-92.

11) Thomsen, L., 1986, Weak elastic anisotropy:

Geophysics, 51, 1954-1966 